\title{
The Visual Order Construction of the Design of the Visual Guide System in the Hotel Environment and Its Value to the Hotel Management
}

\author{
Shubai Li \\ Art College of Xi'an University, 710065
}

Keywords: Hotel environment; Guide; Visual communication; Hotel management

\begin{abstract}
Today, in the rapid development of science and technology, people have a deeper understanding of themselves. From the study of the psychology of the way of thinking to the design of the guidance system for road-seeking behavior, it is hoped that the harmonious relationship between environment and people and architecture can be solved scientifically. The visual order construction of the guidance system is like the design of the spatial catalog in the building environment, which plays an important role in guiding, instructing and evacuating people. The design of the Guidance System in hotel environment is of great practical value in improving the overall management level.

Under the background of the New Era, China's urban construction is developing rapidly, which is influenced by various architectural styles and ideological trends of the modern industrial revolution in the West, and the domestic architecture landscape and interior design industry appear to be quite different. Hotel Industry is a city window industry, often represents the whole image of the city. In order to make the hotel's overall image more contemporary and regional, it is more convenient and comfortable to use the function, the scientific and humanized characteristic hotel is the new trend of contemporary hotel design.
\end{abstract}

\section{The Visual Order Principle of the Guidance System in the Hotel Environment}

The visual order construction of the Guidance System in the hotel environment is like the"Global Positioning System" in the hotel, the design of the guidance system must be based on the characteristics of hotel building space and the scientific grading arrangement and arrangement of People's general thinking habits and behavior patterns, its visual graphic symbols and text must have a coherent and rigorous logical relationship. The following principles must be followed in the construction of the visual order of the hotel guidance system:

The guiding principle of grading,

Through the hotel building characteristics and the operation mode concept fully understand, will guide the visual system logo, according to certain order level from outside to inside, from the big to the small, according to the sequence to carry on the design construction. It is usually defined from the most extended visual effects and functional orientation, such as road signs on public roads, exterior wall interfaces and logo, Logo Towers on the top of the building; The secondary guide is a step-by-step progression from the outside to the specific location and function, such as the hotel parking lot and hotel public area, so that guests enter the hotel, according to the division of function area. The third level guide is the direction guide of the final destination, such as toilet, Lavatory, business center, Conference Room, dining room, guest room, Swimming Pool, etc Finally, it is the expository television system, that is, the four-level guidance system, which mainly embodies the expository and warm-prompting guide, such as the precious animal and plant name, the Prohibition of smoking, the careful falling of water and so on.

\section{The principle of the right place at the right time.}

At the right time and in the right place, is the principle of the right place at the right time. First of all, we should understand and master the characteristics of hotel building space. To determine where hotel guests are prone to confusion, this confusing place is the information node in the hotel environment, which also carries information from both time and space. Before setting up the marking node, it is necessary to find the exact node position, to find and observe the location of the 
node from the angle of hotel building space, in order to complete the system layout of the whole guide logo.

\section{The principle of simplicity and understand ability.}

In the design of the identify modeling, we should not only take into account with the hotel enterprise culture characteristic, but must carry on the understanding to the hotel guest's position. For information to be concise, graphic symbols and materials to be set up with certain coherence, visual identification set logic relationship clear and successive, whether it is old people, children or disabled people or international friends, can be correctly and quickly guide of the right direction, so as to obtain from the hotel environment design to the guest's thoughtfulness and solicitude

\section{The principle of safety, comfort, beauty.}

The hotel industry is the living room of the city. With the rapid development of economy, consumers' ideas and aesthetic consciousness are increasing day by day. The hotel has not only decorated, displayed and constructed the interior interface, but is more inclined to the safety, comfort and emotional experience of the space, and pays attention to the spiritual enjoyment of the people, it is a big trend in modern hotel environment design. In the material and detail treatment, each physical form should strengthen the guest's personal experience and feeling, in the direction of the height of the guide sign materials, lighting design should be combined with the scientific research mode of human-machine engineering, by creating and rendering the atmosphere of the hotel's different functional spaces, the guest experiences the unique design style, the space form and the culture atmosphere.

\section{The Goal and Idea of Visual Order Construction of Hotel Guide System — Taking Anaman Hotel in Hangzhou as an Example.}

\section{Shape and Function}

In the hotel environment, the guide design pursues the effective use of space and completes the accurate communication of information. In the design of the design of the Guide logo, it can not be too conspicuous, but needs to be coordinated into the environment. To beautify and weaken its shape and strengthen the embodiment of its function.

The design factors include:size, appearance, color and material, and the way of presentation. In the appearance design, we should not only distinguish from the environment and highlight the logo, but also integrate with the environment. So the design of the system is to deal with the relationship with the surrounding environment.

The French cloud Anaman Hotel, a well-known themed five-star Hotel near Ling yin Temple, is located as an"18th-century Chinese village" as its architectural setting, in the form of ancient village dwellings, introverted and introverted, full of the casual and elegant oriental temperament Bamboo trees lined the road as if they were in a world away.

The hotel occupies an area of about 14 hectares and has 47 rooms, each with its own courtyard, and maintains the original appearance of the village in the appearance of the hotel. All the guest rooms in the village are distributed along the 600-metre French cloud path, which is paved with green slabs, which form the link between the courtyard of the village. The hotel brings to people the first feeling is quaint and warm, by the stone-paved forest path, play a natural role in the role of natural guidance, its sense of order sublimated without deliberate human suggestion. The stone path is clearly carefully planned and carefully planned, and in detail it seems warm and caring without the cold, hard texture of metal and stone, due to its rational layout and tortuous arrangement.

The hotel's guidance system is designed with a more common vertical and hanging layout, but the wood used for each guide plate is of different quality and size, either on the tree or leaning against the wall or across the Lintel, it's natural, simple, and casual, and it's easy to see. The hotel does not have a door-to-door system, and the guests only give out a key with bamboo decoration. The hotel keeps the floor lights on to the room's population, and even evening guests don't have to worry about getting lost and can't find rooms. The design of the hotel, is a new Chinese style of the natural model works, conveying a kind of indifferent, plain feeling. 


\section{Color and Material.}

The color and material of the signage are important factors in the design of the Guidance System in the hotel environment. In design, it is more important to grasp the recognition. Its color and material must be contrasted with the surrounding environment and in harmony with the surrounding environment. The recognition of the Guide logo must follow the principles of rationality, integrity, systematization and function to the requirements of color and material.

The relationship between the guide color system and the environment color has two kinds:one is to coordinate into the same kind of color or basically consistent, and one is contrast and contrast. The two colors system is used properly, which is the design of success. The value of the presence of a guide is lost if it is hidden in the environment, if it is to be integrated into the environment without being detached from its easy-to-discern utility. The color and material dimension is the sublimation of the order sense of the guidance system in the hotel environment.

\section{Lighting and setting.}

In the lighting configuration of the guiding signs, the first thing to emphasize is the unity of brightness and Hue. In order to achieve the same light source effect during the day and night, the control of light needs to be tested, grasp the refractive and reflection relationship of light and material, and grasp the wavelength of light. In the hotel environment, the use of warm light source can give a warm and intimate feeling. In a relatively stable space environment, the choice of the brightness of light must be appropriate, too bright will destroy the coordination of the environment, too dark to play the role of cue, and should also pay attention to the angle of light source and avoid glare. The safety of the guided visual AIDS on the wall should also be considered.

\section{The Fabric of the Visual Order}

The visual order of the visual order is constructed in the hotel environment. It is necessary to grasp the accuracy, ease of knowledge, rationality and accessibility Guided Visual identification is designed to guide people to find the most convenient, or the best viewing route. At the same time, the visual representation of the Guide logo needs to comply with the design of the design, need to pay attention to a certain artistic nature, these are the focus of the guidance system planning. At the same time, we should pay attention to whether the position and quantity of the logo setting is reasonable. The location of the marking should be accurate and reasonable, and the quantity should not be too much. Too much will affect the harmony of the whole building space environment, and too little will result in the inability to transmit and distinguish direction information, which leads to the failure to reach the destination smoothly, thus losing the meaning of guidance.

\section{The Development of the Visual Order of the Guidance System in the Hotel Environment}

The guidance system is an indispensable way for people to obtain information when they are in unfamiliar environment. As long as you go out, you will face a variety of road-finding problems. From the point of view of space environment design, $\odot$ the designer tries to minimize its existence, and from a practical point of view, he wants to get the convenience from it. The relationship between the contradiction and the shield must coordinate the relationship between the building environment and human beings. Study what kind of environment makes people feel happy, and what kind of environment people get nervous about. The design of the TV system in the future hotel will be closely related to the progress of science and technology, through a large number of research on the design of excellent TV system at home and abroad, digital and interactive design will be the direction of the development of visual order in the Future Hotel Guidance System. Reasonable and effective visual order construction. .

\section{The Hotel Guidance System to Enhance the Value of Hotel Management}

The guidance system is an important part of the public environment of hotel. A well-designed TV system can be used as a language medium, which not only has good guiding function, but also can reflect the deep cultural and artistic background of the hotel. A well-functioning guidance system 
will make it easy for visitors and impress them. In a sense, the guidance system has the latent and undeniable intrinsic value to improve the management level of hotel.

\section{Conclusion}

In the future, the construction of visual order in hotel environment will realize the human-computer interaction of information through the combination of new technology and new material media, such as guided vision system and flat paper medium. With the popularity of devices such as smartphones and tablets, it has become a reality to get accurate and timely information about location. We live in an age of information explosion and Information Without Borders, and the exchange of information on digital interfaces will provide a better platform for obtaining information of all kinds. Non-professional ordinary people can easily access the hotel's various services and information sharing by simply manipulating the visual menu through the windows of their smartphones. As long as the hotel and other service industries and Internet positioning system secure link, the visual order construction of the Guidance System in hotel environment will be more safe, convenient and full of humanistic concern. More and more hotels have realized the value of the guidance system in promoting hotel management, and more and more people, more and more intelligent guide system will soon be applied in the service system of each hotel.

\section{References}

[1]. Bao Shidu, ET AL. :design of Environmental Identification Guidance System, [M] BEIJING:China Construction Industry Press, 2007;

[2] Zhao Yunan: Environmental Information Communication Design, [M] BEIJING: Higher Education Press, 2008;

[3]Hong Xingyu:Logo Design, [M] Wuhan:Hubei Art Press, 2009;

[4]. Xiao Yong. CUI FANGJIAN:"guide design", [M] Wuhan: Hubei Art Press, 2009;

[5]Direction marking system design, [M]Nanchang:Jiangxi Art Press, 2009;

[6]Feng Yi:color design of city TV system, [ m] BEIJING: China Forestry Press, 2012 [7]Xiao Yong, Liang Qingxin: "Look! The design of the Guidance System", [M] Beijing:Thee Publishing House of Electronics Industry, 2013; 